\title{
Reversible Lane Scheme Selection Model Based on the BPR Function and Environmental Benefits
}

\author{
Jiarong Wei ${ }^{1, \text { a) }}$, Liying $\mathrm{Wei}^{1, \text { b) }}$ and Yufeng Cui ${ }^{1, \mathrm{c})}$ \\ ${ }^{1}$ School of traffic and transportation, Beijing JiaoTong University, Beijing 100044, China. \\ a)790827856@qq.com \\ b)lywei@bjtu.edu.cn \\ c)15120800@bjtu.edu.cn
}

\begin{abstract}
By considering the problem of unbalanced tidal traffic flow on the road, reversible lane schemes were given. A reversible lane scheme selection model, which based on the BPR function, was formulated with decision variable of the number of reversible lanes and based on smallest energy consumption and emission costs with the consideration of delay, as well as solution algorithm. The influence of delay reversible lane scheme selection and energy consumption and emission costs were considered in the formulation of model. Using Ronghua Road as a case with simulation software VISSIM, model was verified and the environmental benefits of reversible lane scheme was discussed. The feasibility of model was verified by comparing the results between model calculation and simulation, and the reasonable scheme of reversible lane was calculated. The simulation results show that the delay has been reduced by $25.3 \mathrm{~s}$, and the costs of energy consumption and emission have been reduced by $44.6 \%$ after the implementation of reversible lane scheme considering environmental benefits. It also certifies that not only the delay is reduced but also the energy consumption and emission costs are reduced after the implementation of reversible lane scheme. Thus the environmental benefits are made.
\end{abstract}

Keywords: reversible lane, Model, BPR function

\section{INTRODUCTION}

With the rapid development of economy and expansion of urban scale, many people choose to work in the center of the city and live in the suburbs. And with the relocation of urban residents, tidal characteristics of traffic flow has become increasingly obvious. Tidal traffic is that the traffic volume in the direction of rush into city are greater than the traffic volume in the direction of out of city in the morning peak, and on the contrary in the evening peak. Tidal traffic is a traffic problem in many cities. It makes the traffic flow in both direction of the road is not balanced, one of the direction is traffic congestion and the other direction is idle, resulting in waste of road resources.

In order to solve these problems, the reversible lane technology is introduced as an effective alternative. The reversible lane technology are widely regarded as one of the most cost-effective methods to increase the capacity of an existing roadway. The principle of reversible roadways is to configure the lanes of a roadway to match available capacity to the traffic demand. These roadways are particularly effective because they take advantage of the unused capacity in the minor-flow direction lanes to increase the capacity in the major-flow direction, thereby eliminating the need to construct additional lanes.

A lot of scholars put a great focus on research of reversible lane technical. Foreign scholars were dedicated to studying the practical applications for reversible lane: B. Wolshon studied the benefits and impacts of the reversible lane by using specific examples [1]; In order to eliminate conflict points between reversible lane and intersection, the optimization model of evacuation network based on the reversible lane is constructed [2]. H. Matthew used a bilevel programming model to study the control system of optimal configuration of reversible lane, and verified that the reversible lane can increase the maximum traffic capacity of the road [3]. A. Golub studied practical operation 
results of reversible lane according to the actual cases [4]. The research about reversible lane in China mainly include: Y. Cui studied the traffic organization of Beijing Chaoyang Road reversible lane [5]; X. Y. Gong proposed the traffic direction changing algorithm of traffic volume prediction for urban tidal traffic situation [6]; B. L. Han set up a reversible lanes number adjustment model based on the smallest delay cost [7]; W. H. Zhang proposed the evaluation model of reversible lane operation effect [8].

For study on reversible lane, the foreign scholars put focus on the practical applications, and the domestic scholars mainly put focus on traffic direction changing, traffic organization optimization and reversible lanes number adjustment. While there were a lot of people doing these research, and a few scholars focused on the research about the environmental benefits of reversible lane schemes. And also in the existing study on reversible lane schemes, the environmental benefits of reversible lane were not considered. So in this paper, the reversible lane scheme selection model is studied, which based on smallest energy consumption and emissions costs. The results of the study can reflect the contribution of reversible lane scheme in the mitigation of tidal congestion and environmental pollution.

\section{THE DESCRIPTION OF REVERSIBLE LANE}

Reversible lane is a kind of traffic organization mode, which can change the traffic direction of road or the traffic type of some lanes during different time periods [9]. The conditions for conducting reversible lane scheme are as follows:(1)there is no hard central separators on the road and the number of road lanes is more than 3;(2)the value of directional distribution factor of the road is more than $2 / 3 ;(3)$ the section saturation of major direction reached 0.8 [11].

\section{The description of reversible lane scheme}

For a road from node $\mathrm{M}$ to node $\mathrm{N}$, if the traffic volume in the direction from node $\mathrm{M}$ to node $\mathrm{N}$ is far greater than that from node $\mathrm{N}$ to node $\mathrm{M}$, it can configure one or several lanes in the direction from node $\mathrm{N}$ to node $\mathrm{M}$ to match available capacity to the traffic demand in the opposite direction. The theory of reversible lane scheme is to determine the number of reversible lanes. Taking a road with six-lane as an example, the reversible lane schemes which adjusting one and two lanes are given in Fig.1 (The study excludes the scheme of adjusting 3 lanes, which is one-way traffic).
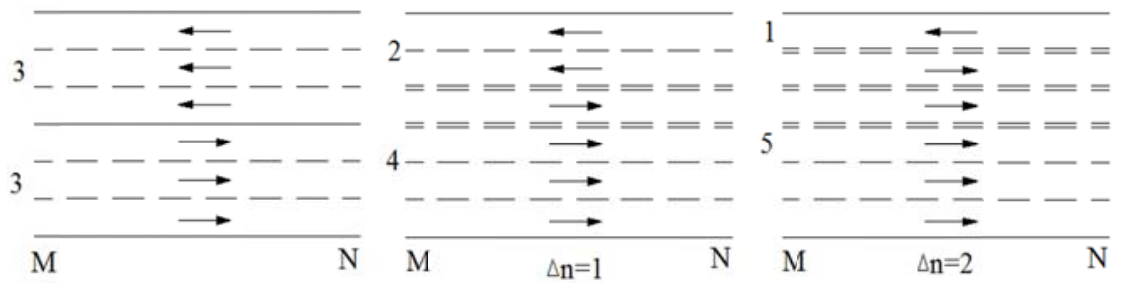

FIGURE 1. Schematic diagram of variable lane schemes for six-lane road.

The road is assumed that the original number of lanes in major and minor direction are $n$ and $n$, respectively, and the number of reversible lane adjusting is $\Delta n$. So the reversible lane schemes of the road are shown in Tab.1. For example, scheme (1) is $\Delta n=1$, which the number of reversible lane is one. So are the rest schemes.

TABLE 1. Reversible lane schemes.

\begin{tabular}{cccc}
\hline $\begin{array}{c}\text { The number of } \\
\text { lane }\end{array}$ & Schemes & Major direction & Minor direction \\
\hline & 1 & $n+1$ & $n^{\prime}-1$ \\
$n+n^{\prime}$ & 2 & $n+2$ & $n^{\prime}-2$ \\
& $\cdots$ & $\ldots$ & $\cdots$ \\
& $n$ & $n+\Delta \mathrm{n}$ & $n^{\prime}-\Delta \mathrm{n}$ \\
\hline
\end{tabular}




\section{Scheme evaluation index}

The tidal traffic has results in environmental costs associated with vehicle delay, and energy consumption and emissions produced by vehicles for each reversible lane scheme are different. Therefore, vehicle delay and energy consumption and emissions should be considered so as to choose the optimal scheme.

\section{Delay}

Road impedance function, also known as volume-delay function, is an expression used to calculate the travel time along a road section. This function reflects the impact of congestion on travel time. Among all the road impedance function, the BPR function developed by FHWA is most representative and most commonly used. The $\mathrm{BPR}$ function is expressed as:

$$
t=t_{0}\left[1+\alpha\left(\frac{V}{C}\right)\right]^{\beta}
$$

Where $t$ is travel time along a road section, $(\mathrm{s}) ; t_{0}$ is the travel time without traffic on the section, (s); $V$ is traffic volume (veh/h) and $C$ is the capacity of the road section, (veh/h); $\alpha, \beta$ are parameters. As for urban roads, the default values are $\alpha=0.15$ and $\beta=0.4$.

The delay $t_{d}$ is the travel time minus the time of free flow $t_{0}$, so the formula of delay is expressed as:

$$
t_{d}=t_{0}\left(\frac{V}{C}\right)^{\beta}
$$

The formula of total delay for all vehicles in the two directions is expressed as:

$$
\begin{gathered}
t_{d}=t_{0}\left(\frac{V_{a}}{C_{a}}\right)^{\beta}+t_{0}\left(\frac{V_{a}^{\prime}}{C_{a}{ }^{\prime}}\right)^{\beta} \\
C_{a}=n c_{a}+\Delta n c_{a} \\
C_{a}{ }^{\prime}=n^{\prime} c_{a}^{\prime}-\Delta n c_{a}{ }^{\prime} \\
V_{a}<C_{a} \\
V_{a}^{\prime}<C_{a}{ }^{\prime}
\end{gathered}
$$

Where: variables for major and minor direction are labeled with $a$ and $a$ ', respectively; $n$ and $n$ ' are the original number of lanes in major and minor direction; $C_{a}$ and $C_{a}$ ' are the total capacity of major and minor direction; $c_{a}$ and $c_{a}$ ' are the single lane capacity of major and minor direction; $V_{a}$ and $V_{a}$ ' are the traffic volume for major and minor direction; $\Delta n$ represents the number of lanes adjusted. The meaning of other variables are the same as defined above. In order to make $\Delta n$ as a decision variables into the formula of delay, the road capacity is simplified as the sum of single lane capacity in formula (4) and (5).

\section{The costs of energy consumption and emissions}

Considering that the energy consumption of vehicles with different emissions are different and the proportion of vehicles with different emissions on the road is also different, so the function of energy consumption and emissions costs can be established based on them. Energy consumption $\operatorname{costs}\left(U_{L}\right)$ is defined as the product of the vehicle energy consumption and the energy price $\left(N_{L}\right)$, and the energy consumption is defined as the product of the vehicle hourly energy consumption $\left(L_{i}\right)$ and the travel time $(T)$ on the road. The costs of treating emissions $\left(U_{E}\right)$ is defined as the product of the volume of vehicle emissions and the price of treating emissions per unit $\left(N_{E}\right)$, and the volume of vehicle emissions is defined as the product of the volume of hourly emissions $\left(E_{i}\right)$ and the travel time $(T)$ on the road. Thus, the energy consumption costs function and the emissions costs function are:

$$
\begin{gathered}
U_{L}=\sum_{i=1}^{5} \alpha_{i} \beta_{i} L_{i} T N_{L} \\
U_{E}=\sum_{i}^{5} \beta_{i} E_{i} T N_{E} \\
T=t_{0}+t_{d}
\end{gathered}
$$


Where $\beta_{1}, \beta_{2}, \beta_{3}, \beta_{4}$, and $\beta_{5}$ are the proportion of the micro car, ordinary car, intermediate car, mid and highgrade car, advanced car on the road, respectively; $\alpha_{i}$ represents the correlation coefficient between emissions and energy consumption of cars; $L_{i}$ represents the hourly energy consumption $(\mathrm{L} / \mathrm{h}) ; E_{i}$ represents the volume of hourly emissions $(\mathrm{L} / \mathrm{h}) ; N_{L}$ represents the price of energy $(\mathrm{RMB} / \mathrm{L}) ; N_{E}$ represents the price of treating emissions per unit $(\mathrm{RMB} / \mathrm{L}), T$ represents the travel time on the road (s).

\section{THE MODEL OF REVERSIBLE LANE SCHEMES SELECTION}

The reversible lane schemes selection model is established based on the function of energy consumption and emissions costs. The model is formulated with decision variable of the number of reversible lanes. The energy consumption and emissions costs is the objective variable for evaluating the efficiency of the scheme. The objective function is:

$$
\min U=U_{L}+U_{E}
$$

Where: $U$ represents the costs of energy consumption and emissions (RMB).

The capacity of the road must meet the traffic demand in both major and minor direction. The bounds for it is:

$$
\begin{aligned}
V_{a}<C_{a} \\
V_{a}{ }^{\prime}<C_{a},
\end{aligned}
$$

The number of reversible lanes is not more than the number of original lanes in both major and minor direction. The bounds for it is:

$$
\begin{gathered}
0<\Delta n<n \\
0<\Delta n<n
\end{gathered},
$$

\section{THE SOLUTION PROCEDURES OF THE MODEL}

Step 1: According to the number of road lanes, all reversible lane schemes for the road are given.

Step 2: Judging whether the present situation of the road satisfy the following three conditions: (1)there is no hard central separators on the road and the number of road lanes is more than 3 ; (2)the value of directional distribution factor is more than $2 / 3$; (3) the section saturation of major direction reached 0.8 .

Step 3: If the above conditions are satisfied, the value of $\Delta n$ will be selected, otherwise the schemes selection of reversible lane will not be carried out.

Step 4: After selecting of reversible lane schemes, determining whether the capacity of major and minor directions meet the actual traffic demand after conducting reversible lane scheme according to the formulas from (4) to (7). If the road capacity of two directions meet the actual traffic demand, then go on the next step, or return to step3. If the above conditions fail to hold for all schemes, the reversible lane will not be set.

Step 5: Using formulas from (3) to (10) to calculate delay and the costs of energy consumption and emissions, and then compare the calculation results of each scheme so as to choose the optimal scheme, which has smallest energy consumption and emissions costs.

\section{CASE STUDY AND SIMULATION}

Using Ronghua Road in Beijing as an example to test the feasibility of the model and assess the effects of reversible lane scheme. Ronghua Road is an urban arterial with six-lane, the design speed of $60 \mathrm{~km} / \mathrm{h}$ and the actual single lane capacity is $1400 \mathrm{veh} / \mathrm{h}$. The traffic volume in major and minor direction in the morning peak are 3700 $\mathrm{veh} / \mathrm{h}$ and $1600 \mathrm{veh} / \mathrm{h}$, respectively.

\section{Reversible Lane Schemes}

There are two reversible lane schemes $(\Delta n=1$ and $\Delta n=2)$ for Ronghua Road according to Tab.1. The present situation of the road satisfy the following three conditions: there is no hard medial strip on the road (in Fig.2) and the number of road lanes is more than 3 ; the directional distribution coefficient is 0.68 (greater than 2/3); the section saturation of major direction reaches 0.8 . 


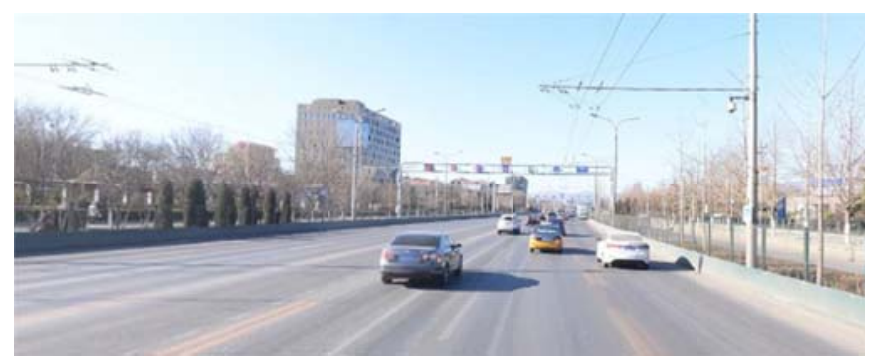

FIGURE 2. Ronghua road in Beijing.

\section{Scheme Selection}

The calculation results of the model for reversible lane schemes are shown in Tab.2. As can be seen from Tab. 2, the optimal reversible lane scheme is $\Delta n=1$, which has minimum delay and smallest energy consumption and emissions costs.

TABLE 2. Calculation results of the model.

\begin{tabular}{cccc}
\hline$\Delta n$ & Delay(s) & $\begin{array}{c}\text { The costs of energy } \\
\text { consumption and } \\
\text { emissions of delay(RMB) }\end{array}$ & $\begin{array}{c}\text { The total costs of energy } \\
\text { consumption } \\
\text { and emissions(RMB) }\end{array}$ \\
\hline present & 54.5 & 210.2 & 557.3 \\
$\Delta n=1$ & 23.8 & 91.8 & 438.8 \\
$\Delta n=2$ & 156.3 & 602.8 & 949.8 \\
\hline
\end{tabular}

The value of each parameter is given as follows: according to the China automobile new network statistics, the values of $\beta_{1}, \beta_{2}, \beta_{3}, \beta_{4}$, and $\beta_{5}$ are $0.251,0.413,0.25,0.05,0.036$, respectively; the value of $\alpha_{i}$ is 0.83 [14]; the value of $L_{i}$ and $E_{i}$ can be known from automobile literature; the value of $N_{L}$ is $5.4(\mathrm{RMB} / \mathrm{L})$, which is the average price of different types of gasoline nearly a year; the value of $N_{E}$ is $2.4(\mathrm{RMB} / \mathrm{L})$ [14].

In formula (11), when $T=t_{d}$, the model calculation result is the costs of energy consumption and emissions caused by vehicle delay; when $T=t_{0}+t_{d}$, the result is the total costs of energy consumption and emissions. As can seen from table 2 , the results comparison between road present situation and scheme $\Delta n=1$ show that delay is reduced by $30.3 \mathrm{~s}$, the costs of energy consumption and emissions produced by delay are reduced by $56.3 \%$ and the total costs of energy consumption and emissions are reduced by $21.3 \%$.

\section{Simulation Evaluation}

To test the feasibility of the model, a micro-simulation was created in VISSIM software. The simulation parameters are set as follow: the simulation time is one hour; every $5 \mathrm{~min}$ is a period of simulation; the vehicle speed distribution is range from $30 \mathrm{~km} / \mathrm{h}$ to $50 \mathrm{~km} / \mathrm{h}$; the simulation times are 3 . The simulation interface is shown in Fig. 3 .

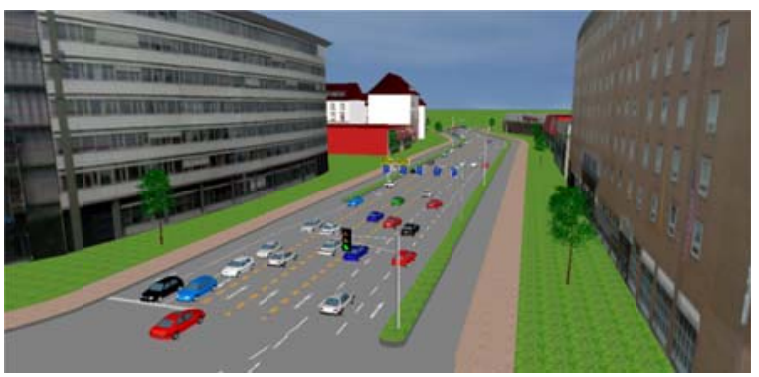

FIGURE 3. Simulation interface of reversible lane scheme for Ronghua Road. 
The before-and-after comparison analysis is conducted in VISSIM to test the feasibility of the model and assess the effects of reversible lane scheme. Tab.3 shows the simulation results. As can be seen from Tab. 3, the optimal reversible lane scheme is $\Delta n=1$, which has minimum delay and smallest energy consumption and emissions costs.

TABLE 3. Simulation results of reversible lane schemes.

\begin{tabular}{|c|c|c|c|}
\hline$\Delta n$ & Delay(s) & $\begin{array}{l}\text { The costs of energy } \\
\text { consumption and } \\
\text { emissions of delay(RMB) }\end{array}$ & $\begin{array}{c}\text { The total costs of energy } \\
\text { consumption } \\
\text { and emissions(RMB) }\end{array}$ \\
\hline present & 56.7 & 218.7 & 565.8 \\
\hline$\Delta n=1$ & 31.4 & 121.1 & 468.4 \\
\hline$\Delta n=2$ & 164.5 & 640.7 & 981.5 \\
\hline
\end{tabular}

The model calculation results in Tab.4 show that the delay is reduced by $30.4 \mathrm{~s}$, the costs of energy consumption and emissions caused by delay are reduced by $56.3 \%$, and the total costs of energy consumption and emissions are reduced by $21.3 \%$ after the implementation of reversible lane scheme $\Delta n=1$.

The simulation results in Tab.4 show that the delay is reduced by $25.3 \mathrm{~s}$, the costs of energy consumption and emissions caused by delay are reduced by $44.6 \%$, and the total costs of energy consumption and emissions are reduced by $17.2 \%$ after the implementation of reversible lane scheme $\Delta n=1$.

Both the model calculation results and the simulation results show that the optimal scheme is $\Delta n=1$ and both the delay and total costs of energy consumption and emissions are reduced after the implementation of reversible lane scheme. So the model of reversible lane schemes selection has certain feasibility, which is proved accurate by simulation results.

TABLE 4. Comparison of improvement results between model calculation and simulation.

\begin{tabular}{cccc}
\hline Improvement & Delay(s) & $\begin{array}{c}\text { The costs of energy } \\
\text { consumption and } \\
\text { emissions of delay(RMB) }\end{array}$ & $\begin{array}{c}\text { The total costs of energy } \\
\text { consumption } \\
\text { and emissions(RMB) }\end{array}$ \\
\hline Model & 30.3 & $56.3 \%$ & $21.3 \%$ \\
Simulation & 25.3 & $44.6 \%$ & $17.2 \%$ \\
\hline
\end{tabular}

To assess the effects of reversible lane scheme, the measure selected for comparison is delay. Tab.5 shows the value of vehicle delay before-and-after the implementation of optimal reversible lane scheme. Fig.4 illustrates the changes of average vehicle delay before-and-after the implementation of the reversible lane scheme.

It can be seen from Tab.5 that the delay of major direction is reduced from $41.5 \mathrm{~s}$ to $16.3 \mathrm{~s}(56 \%)$ after conducting the optimal reversible lane scheme, and the delay of minor direction is changed a little. It also can be seen from Fig.4 that the average vehicle delay in major direction has decreased apparently after the implementation of the reversible lane scheme in the conditions of not balanced traffic volume, and the average vehicle delay of minor direction increased but slightly. The improvement in operation indicates that the reversible lane scheme is an option for solving tidal traffic problems. Therefore, the costs of energy consumption and emission also reduced, it shows that reversible lane scheme has also made environmental benefits.

TABLE 5. Comparison of delay before and after conducting optimal reversible lane scheme.

\begin{tabular}{cccc}
\hline Delay(s) & Major direction & Minor direction & Total \\
\hline before & 41.5 & 15.2 & 56.7 \\
after & 16.3 & 15.1 & 31.4 \\
\hline
\end{tabular}




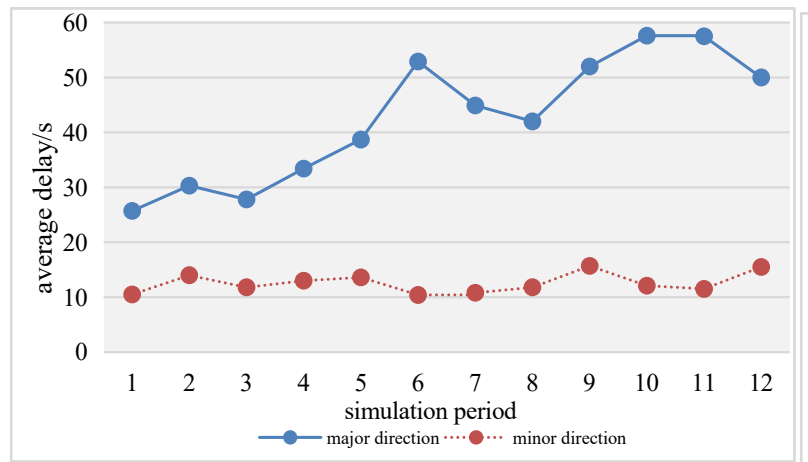

(a) Before

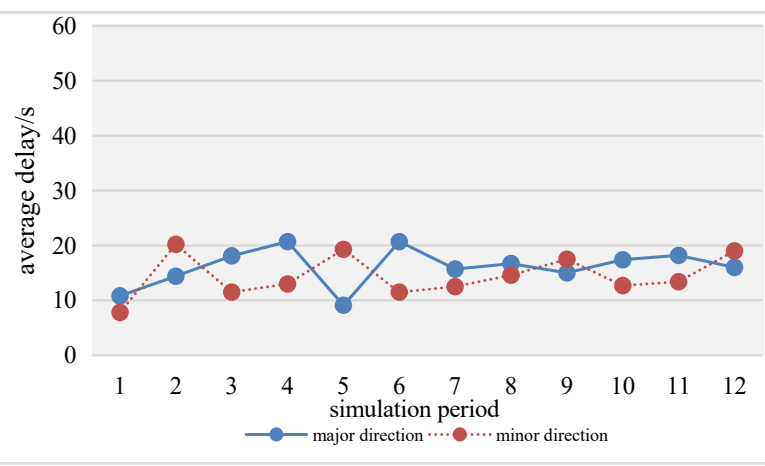

(b) After

FIGURE 4. Comparison of average delay before and after conducting optimal reversible lane scheme.

\section{CONCLUSION}

In this paper, the reversible lane scheme selection model which based on the BPR function was proposed. A mathematical model were formulated with decision variable of the number of reversible lanes. In this model, both vehicle delay and the costs of energy consumption and emissions were taken into account for reversible lane scheme selection. Using Ronghua road to study the feasibility of the model, and micro-simulation tests were also performed. The simulation results confirmed that the model has feasibility, and also showed that both the vehicle delay and the costs of energy consumption and emissions were decreased after the implementation of reversible lane scheme. So the study show that the implementation of reversible lane scheme can obtain environmental benefits.

\section{ACKNOWLEDGMENTS}

This study was conducted with support by the National Natural Science Foundation of China (71101008 and 61473028) and my tutor. Corresponding author at: Traffic Engineering Group, School of Traffic and Transportation, Beijing JiaoTong University; Tel: +86 15801235273; E-mail: 790827856@qq.com.

\section{REFERENCES}

1. B. Wolshon, L. Lambert, Reversible Lane Systems:Synthesis of Practice, Journal of Transportation Engineering, 132(2006)933-944.

2. X. Chi, A. Mark, Lane-based Evacuation Network Optimization: An Integrated Lagrangian Relaxation and Taboo Search Approach, Transportation Research Part C, 19(2011), pp. 40-63.

3. H. Matthew, Dynamic Lane Reversal in Traffic Management, IEEE, Washington, DC, 2011, pp. 1929-1934.

4. A. Golub, Perceived costs and benefits of reversible lanes in phoenix, ITE Journal, 82(2012), pp. 38-42.

5. Y. Cui, D. Liu, Study on reversible lane traffic organization of Beijing ChaoYang Road, Road Traffic and Safety, China, 6(2006), pp. 21-24.

6. X. Y. Gong, S. Kang, Study and Application of Traffic Direction Changing Algorithm for Urban Tidal Traffic Situation, Journal of Transportation Systems Engineering and Information Technology, China, 6(2006), pp. 33-40.

7. B. L. Han, R. Song, and S. W. He, Variable Lanes Number Adjustment Model Based On the Smallest Delay Cost, Road Traffic\&Safety, China, 9(2009), pp. 1-5.

8. W. H. Zhang, G. F. Han, and R. Yan, Evaluation on Effect of Setting Variable Lane in Urban Road Section, Journal of ChongQing JiaoTong University:Natural science Edition, China, 33(2014), pp. 129-133.

9. Z. Z. Yuan, L. Y. Wei, and Y. L. Gu, Road Traffic Management and Control.China: People's Education Publishing Company, 2012, pp. 26-32.

10. Y. Zheng, Y. C. Du, and L. J. Sun, Study On the Road Resistance Function of the Federal Highway Administration, Traffic and Transportation: an academic edition, China, 7(2007), pp. 24-26.

11. X. D. Liang, Q. Guo, and W. P. Yuan, Conversion Opportunity of Reversible Lane Based on BPR Function, Transportation Standardization, China, 2013, pp. 121-124. 
12. G. B. Jia, Z. Z.Yuan Z, Study on Conversion System of Variable Lane Adapted to Tidal Traffic, Highway\&Automotive Applications, China, 11(2013), pp. 55-57.

13. J. Meng, Z. G. Meng, and F. B. Huang, Study on the setting of tidal reversible lane, Road and Traffic Engineering, China, 6(2015), pp. 31-37.

14. X. Y. Guo, The Study of Web-based Spillover Effects Benefits-Case Study of Variable Lane Economic of Beijing, 2011 International Conference on Applied Social Science.Singapore: Advances in Education Research, 2011, pp. 378-382.

15. Z. Bede, T. Peter, The Mathematical Modeling of Reversible Lane System, Periodica Polytechnica: Transportation Engineering, 39(2011), pp. 7-10. 